\title{
SURVEY: 5G WIRELESS COMMUNICATION USING COGNITIVE RADIO
}

\author{
B. Kursheed \\ Research Scholar \\ Department of Electronics and Communication, \\ Visvesvaraya Technological University, Belgavi, Karnataka, India \\ Email: kursheedbellary@gmail.com \\ Dr. Vijayashree R Budyal \\ Department of Mechatronic Engineering, \\ Sri Venkateshwara College of Engineering, Bengaluru, Karnataka, India
}

\begin{abstract}
With the increasing growth in the wireless communication systems, the bandwidth requirement is also continuously increasing, and the increasing demands make the wireless communication resources more and more scarce. Cognitive radio technology facilitates an effective solution for bandwidth scarcity through a process of dynamic and opportunistic spectrum sharing over heterogeneous wireless networks. However, the fundamental goal of the 5G communication system based network is to offer extended coverage, a very high data transfer rate, massive connectivity with minimum latency as performance metrics of $5 \mathrm{G}$ communication system. To achieve these requirements, the $5 \mathrm{G}$ systems exploit the spectrum range from $30 \mathrm{Ghz}$ to $300 \mathrm{GHz}$. This paper presents various aspects of theoretical background that relate the research insights of cognitive radio technology, spectrum sharing, and $5 \mathrm{G}$ communication system. The inferring of the research evolution and trends in the domain of the collaborative study of spectrum sensing, cognitive radio, and $5 \mathrm{G}$ provides future research directions based on the research gap analysis
\end{abstract}

Keywords: 5G Communication; Spectrum Sharing; Cognitive Radio; Energy efficiency; IoT.

\section{Introduction}

The field of wireless communication witnesses its technical advancements in practicing standards from 1st Generation as $1 \mathrm{G}$ to $5 \mathrm{G}$ [1][2]. The respective technological advances include technologies like GSM[3], WCDMA, LTE, WiMAX, MIMO, and mmWaves[4], facilitates voice calls, texting, online data delivery with high speed, and today a massive communication possibilities between anything to anything(X2X) utilizing voice, text, data at very high speed[5]. The overall vision of the future generation communication systems(FGCS) is to achieve high bandwidth, low latency, and more data delivery to offer good broadband services. The 5G evaluation in the current situation eases the communication procedure by following critical technological advancements in its legacy standards. Such critical technologies are Millimeter waves (mm waves), Very large MIMO, Full Duplex-(FDx) system, Beamforming, and Small cells [6]. These key technologies are the enabler for the 5G communication system to optimize the channel capacity by multi-fold compared to the existing $3 \mathrm{G} / 4 \mathrm{G}$ system [7]. Besides, $5 \mathrm{G}$ is a potential communication technology to realize the Internet of Things-(IoT) vision for the massive interconnectivity of multiple devices in the heterogeneous network (HetNet)[8]. Such massive and seamless connectivities in the FGCS exploit spectrum sharing using advanced radio technology, namely cognitive radio (CR)[9]. Therefore, the joint objective of $5 \mathrm{G}$ and $\mathrm{CR}$ based Network (CRN) is to provide a system with the capacity of a high data rate to support the demand of the high traffic. Thus the vision of global connectivity among $\mathrm{X} 2 \mathrm{X}$ gets realized to offer various smart, intelligent, and ubiquitous applications [10]. The figure 1 illustrates a snap-view of the generational evolution of the communication standards. 


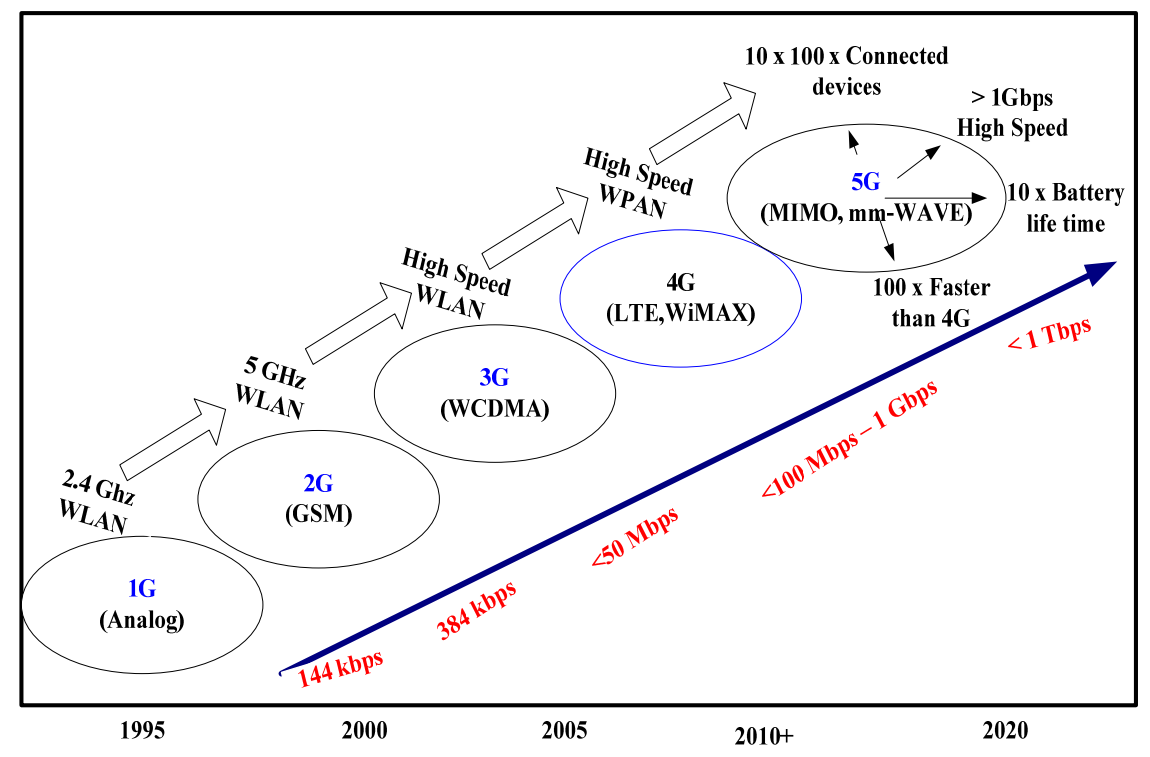

Fig.1. Overviews of different communication technologies

In the last decades, there has been a rapid increment in the spectral resource problem; simultaneously, wireless communication technologies development also emerged as users' demands. In that scenario, the wireless environment unable to satisfy all the user's demands. Hence, Cognitive Radio (CR) technology appeared in response to the spectrum resource limitations, and the primary objective is to increase the spectrum efficiency. The CR provides the possibility to interconnect the existing wireless technologies; also, it offers a complete integrated wireless access network environment. The three essential features of cognitive radio systems are adaptability, cognition and consciousness, and responsiveness[11]. The evolution of CR technology has been defined as a pivotal technology to cope with the shortage of spectrum and minimum usage problems by dynamically reutilize the frequency bands [12], and seems to be full-proof to address the challenges and requirements by which $5 \mathrm{G}$ communication faces [13]. The $\mathrm{CR}$ technology has been given much attention to the information and communication system. The study of Sun et al. [14] provided a comprehensive study of various spectrum sensing algorithms and discussed the pros and cons of respective algorithms. In the survey study of Tanabe et al. [15] have discussed different resource allocation algorithms using CR network techniques and networking architecture. Ahmad et al. [16] overview the recent advancement in the radio resource allocation in CR networks, also discussed the various resource allocation schemes considering the various performance of metrics viz; energy efficiency, QoS assurance, throughput, interference avoidance, and handoff minimization. Another research study Masonta et al. [17], presented a relevant study of spectrum decisions in CR networks based on spectrum features, spectrum selection, and CR reconfiguration. The overlay spectrum access technologies in CR networks and the cooperative and non-cooperative overlay game model CR networks were surveyed [18]. The dynamic spectrum sharing mechanism has discussed by Tehrani et al. [19] also studied prior spectrum sharing schemes with different networking structures, characteristics, use cases, and challenges.

The objective of the proposed study is to offer a detailed survey on spectrum sharing CR technology towards the $5 \mathrm{G}$ system. Despite prior research study on CR, some papers explore the spectrum sharing problems on 5G wireless networks, while different studies distinguish the concept of spectrum sensing [20] and spectrum access in cognitive networks [21]. In [22], Bahaei et al. presented a mechanism of full-duplex and CR technology for future communication systems also focused on research challenges towards $5 \mathrm{G}$ architecture using $\mathrm{CR}$ networking. Compared with existing research, the present survey study providing a comprehensive review of cognitive radio networking scheme to resolve the designing challenges towards $5 \mathrm{G}$ wireless networks. The overall contribution of this survey study is summarized as follows:

- The study describes the spectrum sharing mechanism and spectrum resource management scheme by the utilization of CR technology over $5 \mathrm{G}$ systems, also explores the recent research study towards the spectrum sharing scheme.

- The primary contribution is to provide a comprehensive literature study on CR technology for future cellular network design, including multiple features like more coverage, high data speed, more storage, extensive connectivity, and minimum latency. More specifically, the CR technology may be similar to the $5 \mathrm{G}$, which contains spectrum sharing, spectrum sensing, transmission capability, and spectrum access. 
- The last section highlights the research challenges related to the design and implementation of $5 \mathrm{G}$ networks. It includes parameters like energy efficiency, spectrum sharing, interference mitigation, transmission with the perspective of enhancing both spectrum efficiency and energy efficiency in CR networks.

The structure of this paper is organized as follows: Section-3 illustrates the spectrum sharing mechanism in CR networks, and Section-4 summarizes prior research work on CR in different aspects followed by spectrum management scheme in the $5 \mathrm{G}$ system. Section-5 discusses the evolution of CR technology for future cellular network design. Section-6 overview of the significant similarities between $5 \mathrm{G}$ and CR technologies. Section-7 highlights the research challenges identified in the literature studies. Section- 8 provides the conclusion of the survey study.

\section{Review of Literature}

The spectrum sharing mechanism can increase the spectrum efficiency by connecting multiple nodes using the same spectrum in the same period. The recent, dense implementation of sensor nodes over vast portions of low radio-frequency has been introduced as an essential method of spectrum sharing. Additionally, it is known that the CR spectrum sharing mechanism can use un-used/underuse spectrum temporally and geographically, which improvises the spectral efficiency. In the future wireless communication era $(5 \mathrm{G})$, energy efficiency should be another critical performance parameter, which is motivated by financial as well as environmental considerationshowever, significantly less attention towards current spectral sharing schemes.

New spectrum sharing mechanisms have been introduced to increase the spectrum efficiency by bandwidth exchange also conserves more amount of energy than power control [23]. This mechanism is tightly coupled with cooperative relaying. Another spectral sharing scheme for multi-tier cellular networks, where users can associate with the access point with a shorter transmission range, need significantly less transmitting power. Cognitive radio networks are employed as a promising technology to optimize the energy and remove interference, thus improve the energy as well as spectral efficiency. Various players involve in spectral sharing with different frequencies from different perspectives, which is summarizing in the following figure-2.

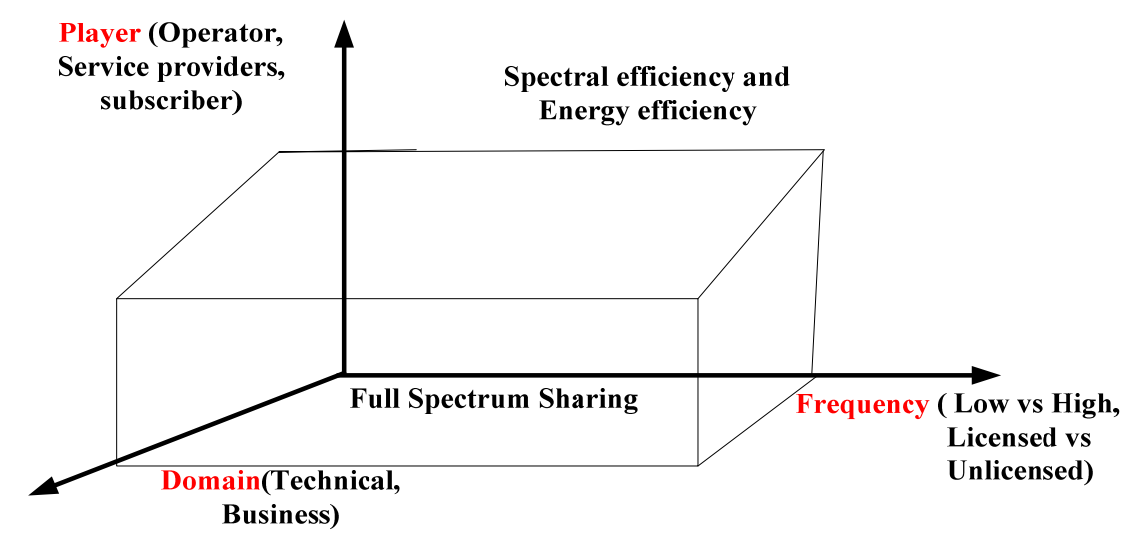

Fig.2. New Spectrum Sharing mechanism in the $5 \mathrm{G}$ era

CR technology is emerging as a critical technology to address the spectrum scarcity problem. Much effort has been made to design a CR network with multiple capabilities, including cognitive cellular, Wi-Fi, and cognitive femto cell.

\section{Spectrum Sharing Mechanism in $\mathbf{C}_{R}$ Networks}

Cognitive Radio $\left(C_{R}\right)$ is an efficient mechanism to expand the spectrum-efficiency and potentially upgrade the spectrum scarcity problems [16]. Generally, $C_{R}$ networks contain two capabilities, including; spectrum sensing and cognitive transmission. In spectrum sensing, cognitive users can sense the radio frequency and collect the spectrum information like; traffic, energy usage, channel gain. While in cognitive transmission, the cognitive users chooses the suitable spectrum band and adapt transmissions based on the collected information.

Cognitive users perform spectrum sensing operation to determine whether current users engage the spectrum band. Two performance metrics are widely utilized to evaluate the accuracy of spectrum sensing, i.e., spectrum detection and false alarm probability. The cooperative spectrum sensing between many cognitive users can be adopted to improve spectrum accuracy. Specifically, cognitive users independently perform the spectrum sensing and take local decisions. Later they deliver the local decisions to the fusion node. Based on local decisions, the fusion node takes the final decision. 
As stated above, the cognitive users select suitable spectrum bands and perform cognitive transmission. It contains two scenarios viz overlay spectrum sharing and underlay spectrum sharing. In overlay spectrum-sharing, there is no sub channel interference among the current users and cognitive users. In underlay spectrum-sharing, cognitive users can cause interference in current users, and cognitive transmission performs for a long duration to satisfy interference constraints. It is clear that from the underlay, spectrum-sharing can achieve good spectrum accuracy and reutilize the spectrum bands by comparing overlay spectrum-sharing in the following figure-3 presenting the typical spectrum sharing scenario over $C_{R}$ networks, where a Macro Base Station (M-BS) and Macro User Equipment (M-UE)represents current macro nodes.

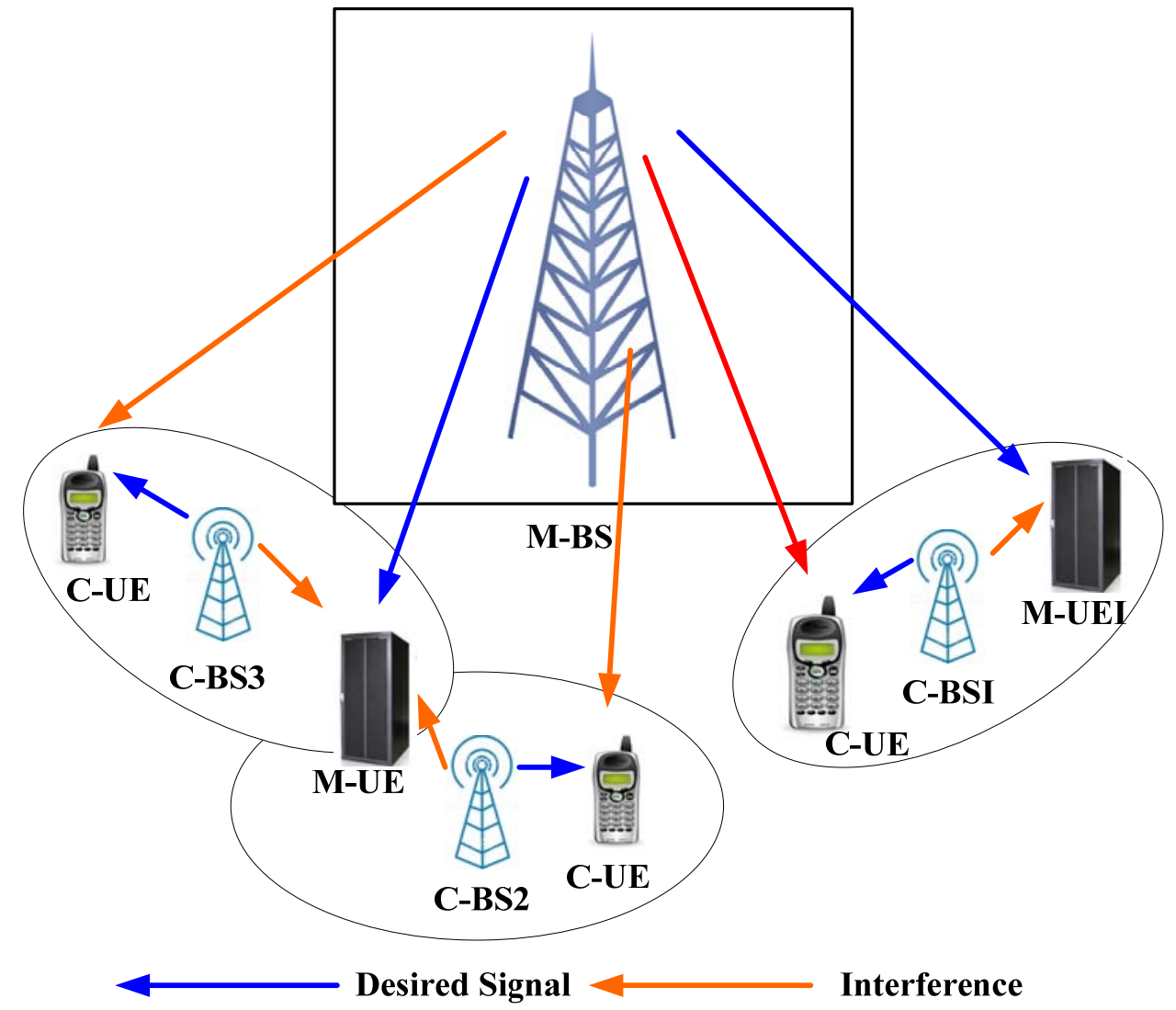

Fig.3. Spectrum sharing mechanism in CR networks

In the above figure 3, there are several cases where downlink transmission may cause interference as an example; the cognitive downlink from cognitive-BS2 to cognitive UE2 and cognitive downlink from cognitive-BS3 to cognitive-UE3 exchange the spectrum bands taken by a current link from M-BS to M-UE2. In that scenario, MUE2 can be interfered with by cognitive-BS2 and cognitive-BS3. Usually, when there are many cognitive downlinks/current downlinks, the energy transmission model design is quite challenging and complicated. In the prior study, the optimal energy transmission model design is obtained in a centralized manner. In the following table-1 summarizing prior research work on CR technology concerning different aspects. 
Table 1 Summary of prior research work on $C_{R}$ in different aspects

\begin{tabular}{|c|c|c|}
\hline \begin{tabular}{l|l} 
Authors & Method \\
\end{tabular} & Descri & \\
\hline Sun et al. [14] & \multirow{2}{*}{$\begin{array}{l}\text { Spectrum } \\
\text { Sensing }\end{array}$} & $\begin{array}{l}\text { Wideband spectrum sensing approach, and use of sub-Nyquist } \\
\text { schemes [14]. }\end{array}$ \\
\hline Yucek et al. [24] & & $\begin{array}{l}\text { Multi-dimensional radio spectrum and transmission process, } \\
\text { different conventional spectrum sensing methods, and cooperative } \\
\text { sensing methods [24]. }\end{array}$ \\
\hline Tanab et al. [15] & \multirow{2}{*}{$\begin{array}{l}\text { Resource } \\
\text { Allocation }\end{array}$} & $\begin{array}{l}\text { Resource allocation for underlay } C_{R} \text { networks, comparative analysis } \\
{[15] \text {. }}\end{array}$ \\
\hline Ahmad et al. [16] & & $\begin{array}{l}\text { Recent advancement in radio resource allocation over } C_{R} \text { networks } \\
\text { [16] }\end{array}$ \\
\hline Masonta et al. [17] & & $\begin{array}{l}\text { Study on spectrum decision mechanism in } C_{R} \text { networks based on } \\
\text { spectrum characterization, selection, and network reconfiguration } \\
\text { [17]. }\end{array}$ \\
\hline Tehrani et al. [19] & \multirow{4}{*}{$\begin{array}{l}\text { Spectrum } \\
\text { Sharing }\end{array}$} & Study of different spectrum sharing schemes for various network \\
\hline Zhang et 1. [25] & & $\begin{array}{l}\text { topologies, challenges in next-generation mobile network } \\
\text { design.[19][25] }\end{array}$ \\
\hline Gand & & Survey of $C_{R}$, full-duplex, and multiple spectrum sharing methods, \\
\hline Höyhtyä et al. [27] & & $\begin{array}{l}\text { spectrum sharing mechanism among satellite telecommunication } \\
\text { and terrestrial networks. [26],[27] }\end{array}$ \\
\hline
\end{tabular}

$\mathrm{C}_{\mathrm{R}}$ technology can reutilize useful spectrum resources without reconfiguring the available spectrum allocation scheme. The primary intention of $\mathrm{CR}$ is to understand the spectrum sharing by dynamic spectrum access. Spectrum sharing implies that secondary users can exploit the idle spectrum of primary users, but only if their communication is undisturbed.

The typical spectrum sharing mechanism involves four steps: A) spectrum-sensing, B) spectrum allocation, C) spectrum access and D). Spectrum handoff.

A) Spectrum sensing: It is the initial phase to ensure the primary users form interference, to perform spectrum sharing, to improvise the spectrum utilization, and realize the different $C_{R}$ applications. Secondary users incessantly detect the spectrum bands utilized by primary users in n-dimensional space (i.e., frequency domain, temporal domain, and spatial domain). The core idea is used to detect whether the primary user appears and determine if the spectrum hole is available. Hence, the precise spectrum holes perception is the initial step in the spectrum sharing.

B) Spectrum allocation: This phase is based on existing spectrum holes and shares the spectrum bands to secondary users. Because the spectrum holes are not fixed, secondary users need to use spectrum holes from the competition while the QoS of secondary-users is varied. Hence, the spectrum holes need to be used efficiently. Spectrum allocation aims to develop efficient spectrum allocation algorithms and policies that can improvise the spectrum efficiency.

C) Spectrum access: The primary user has the priority rights to access spectrum bands, whereas the secondary user behaves as a subordinate relationship. Hence, spectrum access needs an optimal access algorithm to coordinate with multiple secondary users access spectrum holes.

D) Spectrum handoff: The secondary users should switch to the appropriate spectrum if one of the following situations meets. First, when the second user utilizing the available spectrum hole, the appearance of the primary user causes collisions among them. Therefore the secondary user must quit this spectrum band and then switch to another spectrum holes for communication. Secondly, the primary user's geographical location remains constant, while secondary users dynamically change their location, and the remaining spectrum holes of secondary users will be varied, and they require to move to the appropriate spectrum band. Lastly, the spectrum bands utilized by secondary users cannot reach their communication needs; they must move to another spectrum band that can meet their communication requirements. 


\section{Spectrum Management in 5G Networks}

The cloud-based storage solutions provide unlimited memory, with dedicated data processing to sort data and find out the processing signals. These solutions are becoming the benchmark for current massive spectrum sharing and advanced technologies like $\mathrm{CR}$. The wireless radio frequency spectrum is characterized by primary user modeling and spectrum sensing [28]. More clearly, the spectrum management provides a great deal in CR technologies. The dynamic spectrum accessing allows both primary and secondary users to utilize the spectrum slots with their QoS requirements efficiently. The user required $\mathrm{QoS}$ is preserving with their require spectrum mobility for the secondary users in the network, which is named as $5 \mathrm{G}$ networks. Since its mobility features, a secondary user can interchange their position over the cellular network while its transmission and hence, enter into the new network in which targeted radio frequency spectrum slot is already used by the primary user [29].

An efficient spectrum sensing approach provides significant information for which secondary users work. Because traffic in any network and location is time-varying and could not be predicted correctly; therefore, the secondary users contain the actual time information about radio frequency spectrum slots occupancy status to change over the empty slot for resuming its transmission in primary user arrive. In the same way, the spectrum sensing errors are needed to be removed. The primary users exploit their licenses for data transmission according to their QoS requirements. The allocated spectrum bands for wireless networks are underutilized owing to the symbolic customs of mobile applications [30].

The traditional spectrum management schemes are inflexible, where the wireless service provider is assigned a license to operate under a specific spectrum band. It has been very complicated to identify available spectrum bands [31]. Hence, with efficient usage of spectrum creating opportunities, CR networks allow the secondaryusers to allot spectrum slots for their communication and leave it on the primary user [32]. The improvised spectrum management scheme has been introduced by Akhtar et al. [33], which illustrated spectrum utilization policies for cognitive radio networks and contained four significant components, namely, spectrum-sensing, decision, sharing, and mobility framework. The spectrum sharing illustrates how secondary users can select the channel for access coordination. Spectrum mobility enables secondary users to shift to another region while the primary user has arrived. The spectrum can find out the spectrum-holes and quickly find primary user communication on channels occupied by secondary users, while spectrum decision allows the secondary users to select the suitable spectrum slots with QoS [34].

The CRtechnology provides spectrum underutilization and allows secondary users to perform efficiently under ubiquitous wireless networks with high-speed connectivity. The dynamic spectrum allocation mechanism is adapted to resolve the spectrum scarcity problems over future cellular networks. The available spectrum resources can be increased by full-duplex operation in the radio environment so that any node can transmit and receive the radio channel with the same capacity.

Other technologies that enhance futuristic wireless network capacity are three-dimensional (3D) beam forming, Massive MIMO, and mm-Wave communications.

Since visible light communication can support low data rate applications such as location or asset tracking and high data rate applications such as video transmission, it can improve the capacity, efficiency, and security proposed in 5G [35]. The beamforming concept of smart antennas is important in CR to improve the overall spectral efficiency. The main focus of $3 \mathrm{G}$ and $4 \mathrm{G}$ technologies is on mobile broadband users, offering boosted system capacity and higher data rates. Services such as video will also drive the future $5 \mathrm{G}$ technology and support systems with higher system capacity and data rates. Future wireless networks have to provide wireless access to any node or entity that may benefit from being connected. Therefore, $5 \mathrm{G}$ networks are not just improvements to "traditional" mobile broadband technologies. 5G aims at improved support of "machine to machine communication" or "machine-centric communications," also known as the Internet of things. The statistics of North America indicate that their best customers are no longer humans. They are increasingly machines, such as smart utility meters, vehicle infotainment systems, and digital signage [36]. 


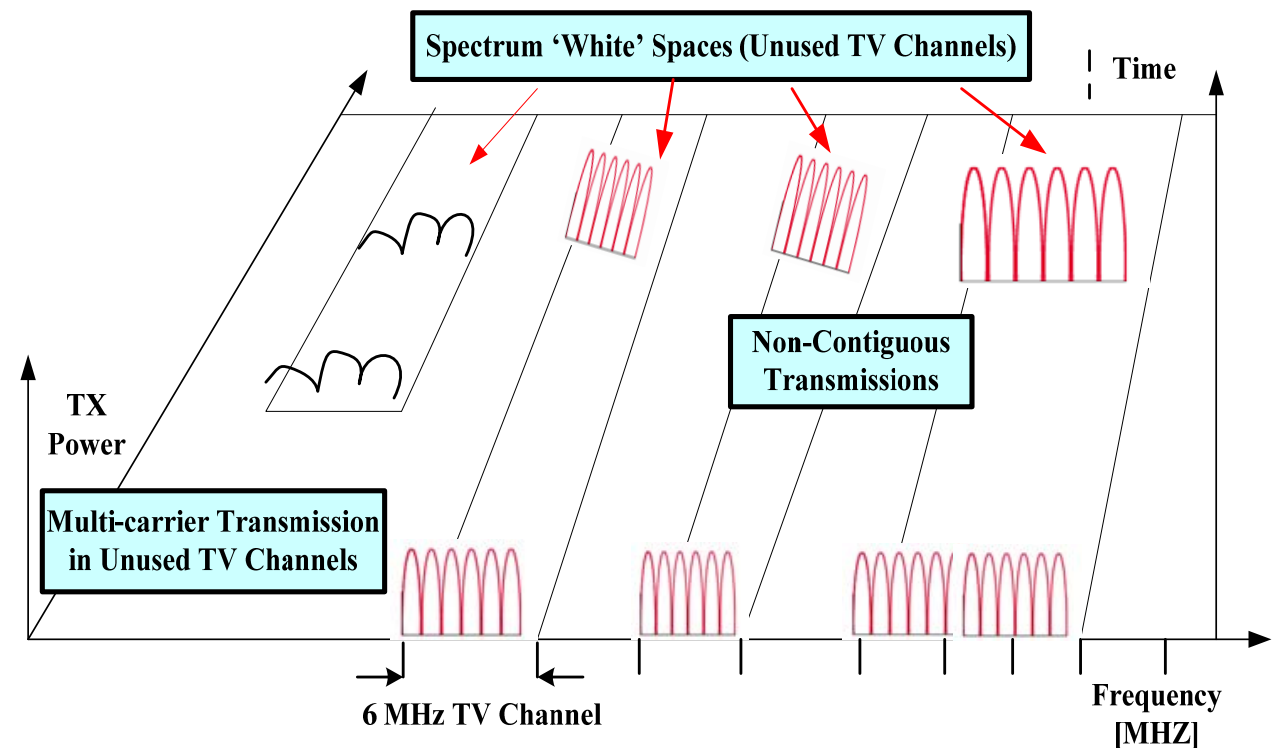

Fig.4. Spectrum management scheme in $\mathrm{C}_{\mathrm{R}}$ networks

\section{Evolution of Cognitive Radio for Future Cellular Networks (5G)}

The appearance of $\mathrm{CR}$ technology has been considered as an efficient mechanism to cope up with spectrum shortage and less resource utilization challenges by dynamically reutilize the spectrum bands assigned to the primary users, and regarded as the perfect solution approach to address the problems and network requirements where the future cellular network may face [12],[13]. Mitola et al. [37] explored the concept of cognitive radio technology. It appeared as potential communication technology to deal with spectrum sensing and spectrum sharing problems with the tremendous growth in wireless networks, especially for future cellular networks, i.e., 5G systems [38]. Zhang et al. [39] have introduced an improved CR technology considering spectrum aggregation and spectrum sharing concept for $5 \mathrm{G}$ cellular networks. This paper represented a new approach which jointly uses the TV white space, licensed Long Term Evolution, time division duplexing bands, and unlicensed spectrum with spectrum sharing and aggregation operations.

Akhtar et al. [40] have discussed the spectrum decision framework and shown the role of cognitive radio technology in the IoT paradigm. Also presented a case study of cognitive radio-based IoT for 5G networks by analyzing spectrum management scheme. The proposed spectrum decision framework weights the frequency band on its performance, occupancy status and guarantees that the proposed investigational study provides a new IoT era to support 5G cellular networks with high data speed. In Shahriar [41], the authors have presented a significant role of the primary user and the secondary-users in the CRnetwork and discussed the overlay and underlay spectrum sharing methodology. Furthermore, it illustrated a CRbased heterogeneous networking concept with its significance and how it can improve the wireless network performance in QoS, coverage, spectral efficiency, data rate, and latency. The cognitive radio-based heterogeneous networking is an emerging wireless technology which has been mainly investigated for future cellular networks, where multiple networks from large cells to small cells, co-exist in the same coverage area [42]. Ghosh et al. [43] have introduced a CRtechnology combined with heterogeneous networks to improve spectrum efficiency.

Exploiting the cognitive radio technology with vertical spectrum sharing mechanism for heterogeneous networks by which fewer priority users in the macro cell region (i.e., secondary users) are handover to the small cell region, and attempting to access the spectrum bands from licensed users (i.e., primary users) in that cell [44].In that case, both primary, as well as secondary-users, can be served by a macro base station. Hence, a spectrum management strategy is essential to manage the spectrum handover operation and mitigate unnecessary handovers and minimize interruptions [45] [46]. The CRbased heterogeneous networks have to gain more attention to resolve such type of problems.

In $5 \mathrm{G}$ communication systems, the available bandwidth is extensive, where there exists a massive number of the non-regular idle spectrum [47]. The channel transmission performance can be enhanced by accessing many nonregular idle spectrums [48]. Liu et al. [48] have introduced the wideband cognitive radio framework for the 5G system using cooperative spectrum sensing method. Through this method can improve the transmitter and receiver performance and avoid the noisy signals. Every cognitive user marks the spectrum availability by doing inverse Fast Fourier Transformation. It also proposed multiple accesses to the CR system to access the noncontinuous idle spectrum. 
The development of smart city wireless networks using radio technology provides more flexibility to the users by interconnecting wireless devices; thus, it provides a complete set of integrated 5G communication networks. In [49], Paulson et al. designed a 5G model using CR technology as a primary applicant and developed a smart city project. The aim was to understand the existing technological concepts for utilizing CR technology in the deployment of $5 \mathrm{G}$ cellular networks. These networks are expected to be more intelligent and efficiently use their network resources. Adigun et al. [50] have reviewed the significance of cognitive radio technology, which has been considered a key enabler for $5 \mathrm{G}$ mobile network deployment. This paper mainly presented the core concept of cognitive radio technology, spectrum optimization mechanism, spectrum aggregation in $5 \mathrm{G}$ networks, and major requirements and challenges for $5 \mathrm{G}$ network design.

In another research study, Sasipriya et al. [51] provided an overview of 5G communication technology and the essential role of cognitive radio in the $5 \mathrm{G}$ system to address the $5 \mathrm{G}$ challenges in the future. Also discussed the need for antennas in CR networks to enhance the $5 \mathrm{G}$ communication performance. The CRnetworks offer multiple services while supporting the wide range of cellular data traffic. The improvement in the network performance can be achieved by integrating multiple network deployment where small cell co-exists with macrocells and performing efficient spectrum sharing operation. The spectrum sharing mechanism can improve spectrum efficiency with minimum energy consumption. An advanced spectrum sharing scheme has been introduced by Yang et al. [52] that saves more energy than power control. The study provided information about the CR and cooperative spectrum sharing methods. It categorized the various spectrum operations and utilization based on a technical and economic perspective also represented a detailed scheme of spectrum flow in $5 \mathrm{G}$ heterogeneous mobile networks, which enhance the spectrum efficiency and energy efficiency.

$\mathrm{Hu}$ et al. [53] provided a comprehensive review of the full-spectrum sharing mechanism over CR based 5G networks. Authors outlined the recent research study of spectrum exploration and presented a taxonomy of cognitive radio-based spectrum sharing by considering four different performance metrics viz; coverage area, transmission capacity, connectivity, and minimum latency. Furthermore, it highlighted the challenges related to the implementation of the cognitive network, including; control channel, and provided detailed research of nonorthogonal multiple access and energy harvesting methods to enhance both spectrum and energy efficiency over cognitive networks. Another investigational study of recent advancements in spectrum sharing techniques for future cellular networks is carried out by Lin et al. [54]. Specifically, the author elaborated on concepts like CRtechnology, full-duplex, and device to device communication, non-orthogonal multiple access, and LTE in an unlicensed spectrum band. For each technique, have presented fundamental characteristics and methodology. By conducting a literature study, the authors emphasized research challenges towards each technique and discussed future scope in the state of spectrum sharing techniques with potential problems.

Hong et al. [55] have presented research progress in the state of the art of CR in 5G networks concerning energy efficiency and spectrum efficiency. In this paper, the authors introduced a different type of network architectures and utilization of cognitive cellular networks, including non-cooperative and cooperative cognitive networks. It also overviewed three levels of spectrum capacity analysis and two performance metrics, which enable a wide range of spectrum efficiency and energy efficiency tradeoff. Jia et al. [56] have discussed various key challenges towards CR applications for future broadband satellite communication systems in 5G. I also designed a cooperative cognitive spectrum sharing algorithm to resolve the mobile user's requirements with the perspective of terrestrial and space segments. In the last, the authors demonstrated a CR based practical scenario using Universal Software Radio Peripheral for a hybrid satellite-terrestrial communication system.

Prasad et al. [57] introduced the "Wireless Innovative System for Dynamic Operating Mega-communication" (WISDOM) concept and provided a new era for future communication networks. This concept brings unlimited wireless global internetworking, more convergence along with numerous multimedia services at high data speed and becomes a strong foundation for $5 \mathrm{G}$ systems. The prime goal was to developed an efficient wireless infrastructure for user-centric communications with a high data rate (i.e., up to 1 terabyte/ps).

In [58], Badoi et al. introduced a combined approach of WISDOM and cognitive radio technology of the 5G communication system. CR technology provides the possibility to interconnect wireless technologies and offers seamless communication with the high data rate. The study described the evolution of cellular communication standards over time and compared the existing communication technologies with 5G systems, also discussed the importance of CR technology in the communication world, as responding to fulfilling the user's requirements. Authors have shown that CR technology is the best solution to implement WISDOM. Prasad et al. [59] have elaborated on the operational concept of WISDOM and shown how this approach is significantly helpful to accomplish the communication requirements. The core idea was to design and implement new technology, network infrastructure that shall universally integrate, interconnect, and provide seamless communication to dynamic users. 


\section{Similarities between $\mathbf{5 G}$ and Cognitive Radio}

After the discussion of current research work on $\mathrm{C}_{\mathrm{R}}$ technology and 5G communication system, it can be observed that there are some significant similarities between them, which are as follows:

- Advanced and flexible technologies.

- Full-spectrum sharing mechanism.

- They are interconnecting with various network resources.

- Both $5 \mathrm{G}$ and $\mathrm{C}_{\mathrm{R}}$ networks have a similar network accessing the feature, i.e., adaptation capability.

- Enhanced and secured physical layer and MAC layer protocols.

- i) An improved smart terminal with the possibility to select the environment, ii) with smart decision capabilities.

- Flexible spectrum resource management that includes efficient data transmission functionality.

- More secure than prior communication technologies.

Hence, it can be noted that the $5 \mathrm{G}$ system aims to interconnect with various communication technologies and the CR networks have the ability to works with wireless technologies.

The CR networks had unique and parallel evolution to that of $5 \mathrm{G}$ systems, is designed and implemented with new additional functionalities such as; i) microprocessor radio controller, ii) digital radio system, iii) software-defined radio, iv) improved CR technology supports all wireless applications, and services and access the information and decision possibilities.

Based on literature studies, the cognitive radio systems must have robust configurations to switch the radio terminals between Direct Sequence Spread Spectrum (DSSS), OFDM, and MAC protocols supported by scalable networks allow the management of reconfigurable radio information.

While 5G systems have global smart terminals that can interconnect with any wireless system, they also can switch over different technologies and provide efficient transmission with QoS.

The QoS comprises multiple parameters, including accessibility, availability, maintainability, etc. As per the requirement of QoS, the CR system must have to select the suitable spectrum band for usage. When mobile users want to collaborate, the operator is responsible for allocating multiple communication channels to the mobile user as per the requirements. The mobile communication system is a dynamic environment where operators have to consider various parameters like communication speed, coverage, handover, and many more, for successful communication establishment.

By considering such types of functionalities, we can say CR technology is the best approach for the all-wireless heterogeneous networks, which is the robust resource management technology and the best solution for 5G system implementation.

\section{Research Challenges}

The 5th generation mobile network uses high-frequency spectrum bands such as millimeter-wave (mm-wave), which contains a high bandwidth range than fewer frequency bands. It can be noted; cognitive users need to scan the wider bandwidth to gather the spectrum band information, which consumes a high amount of energy. The connected challenges include battery terminal problems, particularly for mobile users; usually, they have limited power battery conditions. Therefore, an optimal energy consumption spectrum sharing mechanism is an important requirement in $5 \mathrm{G}$ cellular networks.

$5 \mathrm{G}$ cellular networks are equipped with a wide range of wireless connectivity. More clearly, the cognitive nodes may visible in $5 \mathrm{G}$ networks than current systems because it is unrealistic to reconfigure or manage these nodes in a centralized manner. An efficient interference mitigation scheme is required in current $5 \mathrm{G}$ networks.

The future cellular networks are expected to configure heterogeneously and may be equipped with several wireless technologies, like example; CR, Device-to-Device communication systems, Full-duplex spectrum bands. Similarly, these wireless networks reutilize unlicensed frequency bands. Therefore, it is challenging to assure the performance of LTE transmissions in such complex networks. To accelerate the LTE based spectrum sharing applications, enhanced, flexible wireless technology, spectrum resource management schemes, and network protocols are needed. 


\section{Conclusion}

Cognitive Radio technology is significant to approach to resolve communication-related problems, and it is the fundamental approach to balances the supply and demands in the future communication world. With the resource management of spectrum sharing, the future cellular networks can efficiently accelerate the real-time application and promote the effective development of $5 \mathrm{G}$. In cognitive radio networks, spectrum sharing mechanism is crucial to guarantee that available spectrum bands are assigned to provide the QoS requirements of cognitive radio. In this study, overview the cognitive radio technology based on futuristic challenges and requirements in $5 \mathrm{G}$ cellular networks and comprehensive studies of existing literature. Particularly, the study explored the spectrum sharing mechanism involving four key steps; spectrum-sensing, spectrum allocation, spectrum access, and spectrum handoff. Furthermore, the study presented the literature review on the most recent study in the state of cognitive radio-based 5G system followed by similarities between both $5 \mathrm{G}$ and cognitive radio technology. In the last, have highlighted significant research challenges towards $5 \mathrm{G}$ communication system design, and the proposed survey study is expected to help the researchers to develop an efficient cognitive radio technology for future cellular network implementation.

\section{References}

[1] Goyal J., Singla K., Akashdeep, Singh S. (2020) A Survey of Wireless Communication Technologies from 1G to 5G. In: Smys S., Senjyu T., Lafata P. (eds) Second International Conference on Computer Networks and Communication Technologies. ICCNCT 2019. Lecture Notes on Data Engineering and Communications Technologies, vol 44. Springer, Cham. https://doi.org/10.1007/978-3-030-37051-0_69

[2] J. A. del Peral-Rosado, R. Raulefs, J. A. López-Salcedo and G. Seco-Granados, "Survey of Cellular Mobile Radio Localization Methods: From 1G to 5G," in IEEE Communications Surveys \& Tutorials, vol. 20, no. 2, pp. 1124-1148, Second quarter 2018, doi: 10.1109/COMST.2017.2785181.

[3] K. Holley, "The GSM Short Message Service," IEE Colloquium on GSM and PCN Enhanced Mobile Services, London, UK, 1991, pp. $7 / 1-7 / 5$.

[4] Alexander Kukushkin, "Global System Mobile, GSM, 2G," in Introduction to Mobile Network Engineering: GSM, 3G-WCDMA, LTE and the Road to 5G, Wiley, 2018, pp.59-102, doi: 10.1002/9781119484196.ch7.

[5] H. Ullah, N. Gopalakrishnan Nair, A. Moore, C. Nugent, P. Muschamp and M. Cuevas, "5G Communication: An Overview of Vehicleto-Everything, Drones, and Healthcare Use-Cases," in IEEE Access, vol. 7, pp. 37251-37268, 2019, doi: 10.1109/ACCESS.2019.2905347.

[6] Wang, Cheng-Xiang, Fourat Haider, Xiqi Gao, Xiao-Hu You, Yang Yang, Dongfeng Yuan, Hadi M. Aggoune, Harald Haas, Simon Fletcher, and Erol Hepsaydir. "Cellular architecture and key technologies for 5G wireless communication networks." IEEE communications magazine 52, no. 2 (2014): 122-130.

[7] Y. Ban, C. Li, C. Sim, G. Wu and K. Wong, "4G/5G Multiple Antennas for Future Multi-Mode Smartphone Applications," in IEEE Access, vol. 4, pp. 2981-2988, 2016, doi: 10.1109/ACCESS.2016.2582786.

[8] Rong, Bo, Shuai Han, Michel Kadoch, Xi Chen, and Antonio Jara. "Integration of 5G networks and internet of things for future smart city." (2020).

[9] F. Hu, B. Chen and K. Zhu, "Full Spectrum Sharing in Cognitive Radio Networks Toward 5G: A Survey," in IEEE Access, vol. 6, pp. 15754-15776, 2018, doi: 10.1109/ACCESS.2018.2802450.

[10] S. Borkar and H. Pande, "Application of 5G next generation network to Internet of Things," 2016 International Conference on Internet of Things and Applications (IOTA), Pune, 2016, pp. 443-447, doi: 10.1109/IOTA.2016.7562769.

[11] Peng H, Fujii T. Hybrid overlay/underlay resource allocation for cognitive radio net. in use mobility environment. In: IEEE Vehicular Technology Conference; Las Vegas, USA; Sept. 2013

[12] E. Z. Tragos, S. Zeadally, A. G. Fragkiadakis, and V. A. Siris, “'Spectrum assignment in cognitive radio networks: A comprehensive survey," IEEE Commun. Surveys Tuts., vol. 15, no. 3, pp. 11081135, 3rd Quart., 2013.

[13] P. Rawat, K. D. Singh, and J. M. Bonnin, "Cognitive radio for M2M and Internet of Things: A survey," Comput. Commun., vol. 94, pp. 129, Nov. 2016.

[14] H. Sun, A. Nallanathan, C.-X. Wang, and Y. Chen, " Wideband spectrum sensing for cognitive radio networks: A survey," IEEE Wireless Commun., vol. 20, no. 2, pp. 7481, Apr. 2013.

[15] M. E. Tanab and W. Hamouda, "Resource allocation for underlay cognitive radio networks: A survey," IEEE Commun. Surveys Tuts., vol. 19, no. 2, pp. 12491276, 2nd Quart., 2017.

[16] A. Ahmad, S. Ahmad, M. H. Rehmani, and N. U1 Hassan, "A survey on radio resource allocation in cognitive radio sensor networks," IEEE Communication Surveys Tuts., vol. 17, no. 2, pp. 888917, 2nd Quart., 2015.

[17] M. T. Masonta, M. Mzyece, and N. Ntlatlapa, "Spectrum decision in cognitive radio networks: A survey," IEEE Commun. Surveys Tuts., vol. 15, no. 3, pp. 10881107, 3rd Quart., 2013.

[18] W. Liang, S. X. Ng, and L. Hanzo, “Cooperative overlay spectrum access in cognitive radio networks," IEEE Commun. Surveys Tuts., vol. 19, no. 3, pp. 19241944, 3rd Quart., 2017.

[19] R. H. Tehrani, S. Vahid, D. Triantafyllopoulou, H. Lee, and K. Moessner, 'Licensed spectrum sharing schemes for mobile operators: A survey and outlook," IEEE Commun. Surveys Tuts., vol. 18, no. 4, pp. 25912623, 4th Quart., 2016.

[20] Akyildiz, Ian F., Brandon F. Lo, and Ravikumar Balakrishnan. "Cooperative spectrum sensing in cognitive radio networks: A survey." Physical communication 4, no. 1 (2011): 40-62.

[21] M. Song, C. Xin, Y. Zhao and X. Cheng, "Dynamic spectrum access: from cognitive radio to network radio," in IEEE Wireless Communications, vol. 19, no. 1, pp. 23-29, February 2012.

[22] Shikh-Bahaei, Mohammad, Y-S. Choi, and D. Hong. "Full-duplex and cognitive radio networking for the emerging 5G systems." Wireless Communications and Mobile Computing2018 (2018).

[23] D. Zhang, R. Shinkuma, and N. B. Mandayam, "Bandwidth Exchange: An Energy Conserving Incentive Mechanism for Cooperation," IEEE Trans. Wireless Commun., vol. 9, no. 6, 2010, pp. 2055-65. 
[24] T. Yucek and H. Arslan, "A survey of spectrum sensing algorithms for cognitive radio applications," IEEE Commun. Surveys Tuts., vol. 11, no. 1, pp. 116130, First Quarter 2009.

[25] L. Zhang, M. Xiao, G. Wu, M. Alam, Y.-C. Liang, and S. Li, "A survey of advanced techniques for spectrum sharing in 5G networks," IEEE Wireless Commun., vol. 24, no. 5, pp. 4451, Oct. 2017.

[26] P. Gandotra, R. K. Jha, and S. Jain, “Green communication in next generation cellular networks: A survey," IEEE Access, vol. 5, pp. 1172711758, Jul. 2017.

[27] M. Höyhtyä et al., "Database-assisted spectrum sharing in satellite communications: A survey," IEEE Access, vol. 5, pp. 2532225341, Dec. 2017.

[28] Lee W-Y, Akyildiz I. A spectrum decision framework for cognitive radio networks. IEEE Transactions on Mobile Computing. 2011;10(2):161-174

[29] El Tanab M, Hamouda W. Resource allocation for underlay cognitive radio networks: A survey. IEEE Communication Surveys and Tutorials. 2017;19(2):1249-1276, Secondquarter. DOI: 10.1109/COMST.2016.2631079

[30] Akyildiz IF, Lee W-Y, Vuran MC, Mohanty S. A survey on spectrum management in cognitive radio networks. CSE JournalA articles. 2008:87

[31] Lee W-Y, Akyildiz IF. Spectrum-aware mobility management in cognitive radio cellular networks. IEEE Transactions in Mobile Computing. 2012;11(4):529-542

[32] Bohning D. Notice of Proposed Rule Making and Order. FCC, ET Docket no. 03-222; 2003. pp. 197-200

[33] Akhtar AN, Rashdi A, Arif F. Fusion based spectrum decision framework for cognitive radio users. 2015 IEEE 16th International Symposium on A World of Wireless, Mobile and Multimedia Networks (WoWMoM), Boston, MA, 2015. pp. 1-6.

[34] Masonta MT, Mzyece M, Ntlatlapa N. Spectrum decision in cognitive radio networks: A survey. IEEE Communication Surveys and Tutorials. 19(3):1924-1944

[35] 5G: Challenges, Research Priorities, and Recommendations, NetWorld2020 ETP European Technology Platform for Communications Networks and Services, August 2014.

[36] 4G Americas 5G Spectrum Recommendations August 2015.

[37] J. Mitola and G. Q. Maguire, Jr., "Cognitive radio: Making software radios more personal," IEEE Pers. Commun., vol. 6, no. 4, pp. 1318, Apr. 1999.

[38] X. Hong, J. Wang, C.-X. Wang, and J. Shi, "Cognitive radio in 5G : a perspective on energy-spectral efficiency tradeoff," IEEE Commun. Mag., vol. 52, no. 7, pp. 46-53, Jul. 2014.

[39] Zhang, Wensheng, Cheng-Xiang Wang, Xiaohu Ge, and Yunfei Chen. "Enhanced 5G Cognitive Radio Networks Based on Spectrum Sharing and Spectrum Aggregation." IEEE Transactions on Communications 66, no. 12 (2018): 6304-6316.

[40] Akhtar, Ahmad Naeem, Fahim Arif, and Adil Masood Siddique. "Spectrum Decision Framework to Support Cognitive Radio Based IoT in 5G." In Cognitive Radio in 4G/5G Wireless Communication Systems. IntechOpen, 2018.

[41] Moghaddam, Shahriar Shirvani. "Introductory Chapter: Primary and Secondary Users in Cognitive Radio-Based Wireless Communication Systems." In Cognitive Radio in 4G/5G Wireless Communication Systems. IntechOpen, 2018.

[42] Hong X, Wang J, Wang C-X, Shi J. CR in 5G: A perspective on energy-spectral efficiency tradeoff. IEEE Communications Magazine. 2014;52(7):46-53

[43] Ghosh A, Mangalvedhe H, Ratasuk R. Heterogeneous cellular networks: From theory to practice. IEEE Communications Magazine. 2012;50(6):54-64

[44] Gur G, Bayhan S, Alagz F. Cognitive femtocell networks: An overlay architecture for localized DSA. IEEE Wireless Communications. 2010;17(4):62-70

[45] Habibzadeh A, Shirvani Moghaddam S, Razavizadeh SM, Shirvanimoghaddam M. Spectrum handover mechanism for secondary users in cognitive femtocell HetNets. In: The 24th Iranian Conference on Electrical Engineering; Shiraz, Iran; 2016

[46] Habibzadeh A, Shirvani Moghaddam S, Razavizadeh SM, Shirvanimoghaddam M. Modeling and analysis of traffic-aware spectrum handover schemes in cognitive HetNets. Trans. ETT. Dec. 2017;28(12). DOI: 10.1002/ett.3199.

[47] M. Jia, X. Gu, Q. Guo, W. Xiang, N. Zhang: Broadband Hybrid Satellite-Terrestrial Communication Systems Based on Cognitive Radio Toward 5G. IEEE Wireless Communications, 23 (6) (2016) 96-106.

[48] X. Liu, F. Li, Z. Na, Optimal Resource Allocation in Simultaneous Cooperative Spectrum Sensing and Energy Harvesting for Multichannel Cognitive Radio, IEEE Access 5 (2017) 3801-3812.

[49] Paulson, Eberechukwu \& KAMALUDIN, Mohamad \& KAMILAH, Sharifah \& Dauda, Umar. (2017). Cognitive Radio in 5G - A Smart City Perspective-

[50] Adigun, Olayinka, Mahdi Pirmoradian, and Christos Politis. "Cognitive radio for 5G wireless networks." Fundamentals of 5G Mobile Networks (2015): 149-163.

[51] Sasipriya, S., and R. Vigneshram. "An overview of cognitive radio in 5G wireless communications." In 2016 IEEE International Conference on Computational Intelligence and Computing Research (ICCIC), pp. 1-5. IEEE, 2016.

[52] Yang, Chungang, Jiandong Li, Mohsen Guizani, Alagan Anpalagan, and Maged Elkashlan. "Advanced spectrum sharing in 5G cognitive heterogeneous networks." IEEE Wireless Communications 23, no. 2 (2016): 94-101.

[53] Hu, Feng, Bing Chen, and Kun Zhu. "Full spectrum sharing in cognitive radio networks toward 5G: A survey." IEEE Access 6 (2018): 15754-15776.

[54] Zhang, Lin, Ming Xiao, Gang Wu, Muhammad Alam, Ying-Chang Liang, and Shaoqian Li. "A survey of advanced techniques for spectrum sharing in 5G networks." IEEE Wireless Communications 24, no. 5 (2017): 44-51.

[55] Hong, Xuemin, Jing Wang, Cheng-Xiang Wang, and Jianghong Shi. "Cognitive radio in 5G: a perspective on energy-spectral efficiency tradeoff." IEEE Communications Magazine 52, no. 7 (2014): 46-53.

[56] Jia, Min, Xuemai Gu, Qing Guo, Wei Xiang, and Naitong Zhang. "Broadband hybrid satellite-terrestrial communication systems based on cognitive radio toward 5G." IEEE Wireless Communications 23, no. 6 (2016): 96-106.

[57] Prasad, R., \& Ohmori, S. (2009). Ubiquitous easy life. In the Second Japan EU Symposium on the "Future Internet," Tokyo. (2009). 
[58] Badoi, Cornelia-Ionela, Neeli Prasad, Victor Croitoru, and Ramjee Prasad. "5G based on cognitive radio." Wireless Personal Communications 57, no. 3 (2011): 441-464.

[59] Prasad, Ramjee. 5G: 2020 and Beyond. River Publishers, 2014.

\section{Biography of Authors}

\begin{tabular}{|l|l|}
\hline & $\begin{array}{l}\text { B. Kursheed has completed her B.E in Engineering in Electronics \& Communication } \\
\text { Engineering, M.Tech in Computer networks in 2004 and 2012 respectively from } \\
\text { Visvesvaraya Technological University, Belgavi, Karnataka, India. She is pursuing her } \\
\text { research for the award of a Ph.D. in communication from Visvesvaraya Technological } \\
\text { University, Belgavi, Karnataka. Currently working as an AssistantProfessor-department of } \\
\text { Mechatronics, Sri Venkateshwara College of Engineering, Bengaluru, India. She has 15 } \\
\text { years of rich experience as an academician. She has published her research work into } \\
\text { various International conferences and journals of repute. Her area of interest includes } \\
\text { Wireless Networks, Communication, Cognitive Radio Networks, and Machine Learning. }\end{array}$ \\
\hline Dr. Vijayashree R Budyal, Professor and HOD -Mechatronics, Sri Venkateshwara \\
College of Engineering (SVCE), Holds a Ph.D. degree in Wireless Communication and \\
Networking from Visveswaraya Technological University, Belagavi. She is experienced as \\
an Academician from 25+ years. Area of interest includes Wireless Communication, \\
Wireless Sensor Networks, Multimedia Communication, Mobile Ad hoc Networks, \\
Distributed Mobile Computing, Fuzzy Logic, and Neural Networks and published 10+ \\
papers in various reputed International Journals,19+ papers in various International \\
Conference. Best Research Paper award for "ANFIS and Agent-Based Bandwidth and \\
Delay Aware Anycast Routing in Mobile Ad hoc Networks" by Government of Karnataka \\
Vision Group on Science and Technology Department of Information Technology, \\
Biotechnology, and Science \& Technology VGST, 2013-2014.
\end{tabular}

